\title{
Relationship between body mass index and a novel oxidative stress marker thiol/disulfide homeostasis
}

Gökhan AKKURT ${ }^{1}$, Mustafa ALIMOĞULLARI ${ }^{2}$, Burcu BULUT ${ }^{3}$, Sevgi BiLMEZ ALTAY4 ${ }^{4}$ Didem SUNAY5 ${ }^{5}$ Murat ALIŞIK ${ }^{6}$

\author{
${ }^{1}$ General Surgery Clinic, Health Sciences University, Ankara Numune Training and Research Hospital, Ankara, Turkey \\ ${ }^{2}$ General Surgery Clinic, Health Sciences University, Ankara Keçiören Training and Research Hospital, Ankara, Turkey \\ ${ }^{3}$ Family Medicine Clinic, Bolu Mengen Public Hospital, Bolu, Turkey \\ ${ }^{4}$ Internal Medicine Clinic, Karabük Training and Research Hospital \\ ${ }^{5}$ Family Medicine Clinic Karabük University, Karabük, Turkey \\ ${ }^{6}$ Biochemistry Clinic, Ankara Polatlı Public Hospital, Polatlı, Ankara, Turkey \\ Correspondence \\ Gökhan AKKURT \\ Sağlık Bilimleri Üniversitesi, Ankara Numune Eğitim ve Araştırma Hastanesi, Hacettepe Mahallesi Talatpaşa Bulvarı No:44 Altındağ Ankara, Türkiye \\ e-mail:drakkurt06@gmail.com
}

\begin{abstract}
This study aimed to investigate the relationship between body mass index (BMI) and thiol/disulfide homeostasis, which is used as a novel marker of oxidative stress.

A total of 328 patients admitted to the Family Medicine Polyclinic of Karabük University were included in the study. The demographic characteristics, additional diseases, smoking history, and BMI measurements of the patients were evaluated. Patients with chronic diseases and active malignancy were excluded from the study. The native thiol (sh), total thiol (tt), disulfide (ss), disulfide/native thiol (ss/sh), disulfide/total thiol (ss/tt), and native/total thiol (sh/tt) values of patient's blood samples were analyzed using thiol/disulfide homeostasis kit at the biochemistry laboratory of Yildırım Beyazit University. The patients were divided into four groups according to BMI measurements as follows: BMI <18.5, BMI 18.5-24.9, BMI 25-29.9, and $\mathrm{BMI} \geq 30$. The relationship of patient's thiol values with the BMI and smoking status was statistically analyzed.
\end{abstract}

A negative correlation was found between BMI and both sh and tt values. However, a positive correlation was observed between ss, ss/sh, ss/tt, and sh/tt values and the increase in the BMI. Further, the BMI was found to be an independent variable and statistically significant in all thiol levels except disulfide in the analysis using the general linear model.

It is predicted that thiol/disulfide homeostasis measurements can be widely used in diagnosis because they are cheap and easily applicable and accessible.

Key words: BMI, oxidative stress, thiol/disulfide

\section{INTRODUCTION}

The oxidant-antioxidant balance of the organism should be protected to maintain a healthy life. Free radicals are produced endogenously during the normal metabolic process (1). Free radicals have the potential to damage and interact with the cell components, such as lipids, nucleic acids, and proteins, in the body. Oxidative stress may develop due to the insufficiency of the antioxidant defense system and/or increase in free radical formation in the organism $(2,3)$. It is important to maintain the correct levels of reactive oxygen species (ROS) and reactive nitrogen species in the body. Therefore, many antioxidant systems are ac- tivated to reduce the toxicity of free radicals (4).

Obesity is defined as a chronic disease caused by excess energy consumption. In obese patients, oxygen consumption and mitochondria-induced superoxide, hydroxyl radical, and hydrogen peroxide formation increase due to the increase in mechanical load. The myocardial metabolism in oxidative stress further increases in obesity, especially due to the overproduction of ROS and poor antioxidant system, resulting in protein, lipid, and DNA damage (5). The thiol pool in the plasma is largely composed of low-molecularweight thiols such as albumin, cysteinyl glycine, ho- 
mocysteine, cysteine (Cys), $\gamma$-glutamylcysteine, and glutathione6. Thiols react with oxidants and generally form disulfide bonds in the cell (2). When oxidative stress increases, the oxidation of Cys residues may result in the reversible formation of mixed disulfide bonds between the low-molecular-weight thiols and the protein thiol groups. These disulfide bonds can be reduced back to the thiol groups, and eventually thiol/disulfide homeostasis is maintained (7). Studies have shown that thiol/disulfide homeostasis plays an important role in signal transduction, detoxification, apoptosis, and cellular signal transduction (8). The present study aimed to investigate the relationship between $\mathrm{BMI}$ and thiol/disulfide homeostasis, which is a new marker of oxidative stress.

\section{MATERIALS AND METHODS}

A total of 328 patients admitted to the Family Medicine Polyclinic of Karabük Training and Research Hospital, Turkey, between January 2019 and February 2019 were included in the study.

The demographic characteristics, additional diseases, smoking history (more than 10 cigarettes per day in about 10-20 years), and BMI of the patients were evaluated. Patients with chronic diseases (hypertension, diabetes mellitus, rheumatic diseases, liver failure, and so forth) and patients with active malignancy were excluded from the study.

The blood samples of the participants were put into plain tubes during hospitalization. The serum was taken after the samples were centrifuged at $1500 \mathrm{~g}$ for $10 \mathrm{~min}$ and stored at $-80^{\circ} \mathrm{C}$ until analysis. Thiol/disulfide homeostasis was investigated as described previously. First, reducible disulfide bonds reduced to form free functional thiol groups. Reaction with 5,5'-dithiobis-(2-nitrobenzoic) acid was performed, and all thiol groups were detected. The difference between total and native thiols was calculated, and half of it provided the dynamic disulfide amount (-S-S). Native thiol (-SH) and disulfide amounts (-S-S) were determined. Then, native thiol/disulfide $(-\mathrm{S}-\mathrm{S}-/-\mathrm{SH})$, native thiol/total thiol, and disulfide /total thiol ratios were calculated.

The patients were divided into four groups according to BMI measurements as follows: $\mathrm{BMI}<18.5$, BMI 18.5-24.9, BMI 25-29.9, and BMI $\geq 30$, and also divided into two groups as smokers and nonsmokers. The relationships of patient's native thiol, total thiol, disulfide, disulfide/native thiol, disulfide/total thiol, and native/total thiol values with the BMI and smoking status were statistically analyzed.

Consent was obtained from all patients, with ethical approval from Karabük University (1867350).

\section{Statistical analyses}

Data were analyzed using SPSS for Windows v.15.0 (SPSS,Inc., IL, USA). Descriptive and frequency analyses were performed. A general linear model was used to evaluate independent categorical and numeric variables. The level of statistical significance was set at $P<0.05$.

\section{RESULTS}

Of the 328 patients, $57.3 \%$ were female and $42.7 \%$ were male. The mean age and BMI of the female and male patients were 38.6 and 30.2, respectively. Among admitted patients with BMI measurements, $1.5 \%$ had a BMI $\left(\mathrm{kg} / \mathrm{m}^{2}\right)<18.5,18.9 \%$ a BMI between 19 and $24.9,30.8 \%$ a BMI between 25 and 29.9 , and $48.8 \%$ a $\mathrm{BMI}>30$. Further, $37.2 \%$ of the patients were smokers (Table 1). A negative correlation was found between BMI and native thiol and total thiol values. However a positive correlation was found between the values of disulfide, disulfide/native thiol, disulfide/total thiol, and native/total thiol and the increase in the BMI (Table 2).

The same correlation was also observed in obese

Table 1: General characteristics of patients

\begin{tabular}{lll}
\hline & $\mathrm{N}$ & $\%$ \\
\hline Age, year, mean (range) & $38.6(17-79)$ & \\
\hline Sex & & \\
$\quad$ Male & 140 & 42.7 \\
$\quad$ Female & 188 & 57.3 \\
\hline BMl, kg/m & & \\
$<18.5$ & 5 & 1.5 \\
$19-24.9$ & 62 & 18.9 \\
$25-29.9$ & 101 & 30.8 \\
$>30$ & 160 & 48.8 \\
\hline Smoking & & \\
Smokers & 122 & 37.2 \\
Nonsmokers & 206 & 62.8 \\
\hline
\end{tabular}


Table 2: Mean values according to BMI.

\begin{tabular}{lllll}
\hline & 18.5 & $19-24$ & $25-29$ & 30 \\
\hline Native SH & $413.6 \pm 36.7$ & $388.2 \pm 7.7$ & $359.3 \pm 6.7$ & $314.6 \pm 5.7$ \\
Total thiol & $456.4 \pm 39.4$ & $429.3 \pm 8$ & $405.6 \pm 5.2$ & $356.7 \pm 5.3$ \\
Disulfide SS & $21.3 \pm 2.4$ & $20.5 \pm 0.7$ & $20.0 \pm 0.5$ & $19.0 \pm 0.5$ \\
SSSH & $5.2 \pm 0.5$ & $5.4 \pm 0.2$ & $5.6 \pm 0.1$ & $6.1 \pm 0.1$ \\
SS total thiol & $4.7 \pm 0.4$ & $4.8 \pm 0.1$ & $5.0 \pm 0.1$ & $5.3 \pm 0.1$ \\
SH total thiol & $90.5 \pm 0.8$ & $90.3 \pm 0.3$ & $89.6 \pm 0.3$ & $88.8 \pm 0.3$ \\
Age & $32.0 \pm 6.9$ & $32.2 \pm 1.7$ & $37.4 \pm 1.0$ & $42.1 \pm 1$ \\
Weight & $49 \pm 1.9$ & $63.9 \pm 1.4$ & $75.7 \pm 0.9$ & $90.4 \pm 1.1$ \\
BMI & $17 \pm 0.3$ & $21.8 \pm 0.2$ & $26.8 \pm 0.1$ & $36.1 \pm 0.3$ \\
\hline
\end{tabular}

subgroups (BMI $>30 \mathrm{~kg} / \mathrm{m}^{2}$ ) (Table 3 ). The BMI was found to be an independent variable for all thiol values except disulfide in the analysis with the general linear model. These parameters were not statistically significant for disulfide levels. Age and sex were statistically significant for total thiol and native thiol levels. The BMI had the highest partial eta squared and was found to be the most effective variable for total thiol and native thiol levels (Table 4).

\section{DISCUSSION}

Obesity is a disease with high mortality and morbidity due to health complications. It is characterized by an excessive increase in fat tissue in the body as a result of excess energy consumption. Many chronic diseases such as hypertension (HT), cardiovascular

Table 3: Mean values in obese patients according to BMI.

\begin{tabular}{llll}
\hline & $30-34$ & $35-39$ & 40 \\
\hline Native SH & $335.3 \pm 8.0$ & $308.5 \pm 12.4$ & $280.0 \pm 8.3$ \\
Total thiol & $376.0 \pm 7.1$ & $360.6 \pm 10.2$ & $316.8 \pm 9.1$ \\
Disulfide SS & $18.9 \pm 0.8$ & $20.0 \pm 0.9$ & $18.3 \pm 1.0$ \\
SSSH & $5.7 \pm 0.2$ & $6.3 \pm 0.2$ & $6.6 \pm 0.3$ \\
SS total thiol & $5.1 \pm 0.2$ & $5.5 \pm 0.2$ & $5.7 \pm 0.3$ \\
SH total thiol & $89.1 \pm 0.4$ & $88.5 \pm 0.4$ & $88.4 \pm 0.6$ \\
Age & $41.1 \pm 1.2$ & $45.2 \pm 2.5$ & $41.4 \pm 2.0$ \\
Weight & $88.5 \pm 1.3$ & $92.3 \pm 2.0$ & $103.7 \pm 2.6$ \\
BMI & $32.0 \pm 0.1$ & $37.2 \pm 0.2$ & $43.0 \pm 0.3$ \\
\hline
\end{tabular}

Table 4: Univariant analyses with the general linear model.

\begin{tabular}{|c|c|c|c|c|c|c|c|c|c|c|c|c|}
\hline & \multicolumn{2}{|c|}{$\begin{array}{c}\text { Native } \\
\text { SH }\end{array}$} & \multicolumn{2}{|c|}{$\begin{array}{l}\text { Total } \\
\text { thiol }\end{array}$} & \multicolumn{2}{|c|}{ Disulfide SS } & \multicolumn{2}{|c|}{ SSSH } & \multicolumn{2}{|c|}{$\begin{array}{l}\text { SStotal } \\
\text { thiol }\end{array}$} & \multicolumn{2}{|c|}{$\begin{array}{l}\text { SHtotal } \\
\text { thiol }\end{array}$} \\
\hline & $\begin{array}{c}P \\
\text { value }\end{array}$ & $\begin{array}{c}\text { Partial } \\
\text { eta } \\
\text { squared }\end{array}$ & $\begin{array}{c}P \\
\text { value }\end{array}$ & $\begin{array}{c}\text { Partial } \\
\text { eta } \\
\text { squared }\end{array}$ & $\begin{array}{c}P \\
\text { value }\end{array}$ & $\begin{array}{l}\text { Partial eta } \\
\text { squared }\end{array}$ & $\begin{array}{c}P \\
\text { value }\end{array}$ & $\begin{array}{l}\text { Partial eta } \\
\text { squared }\end{array}$ & $\begin{array}{c}P \\
\text { value }\end{array}$ & $\begin{array}{c}\text { Partial } \\
\text { eta } \\
\text { squared }\end{array}$ & $\begin{array}{c}P \\
\text { value }\end{array}$ & $\begin{array}{c}\text { Partial } \\
\text { eta } \\
\text { squared }\end{array}$ \\
\hline Age & 0.000 & .051 & 0.000 & .070 & 0.125 & .007 & 0.635 & .001 & 0.634 & .001 & 0.570 & .001 \\
\hline BMI & 0.000 & .155 & 0.000 & .210 & 0.065 & .011 & 0.009 & .021 & 0.015 & .018 & 0.014 & .019 \\
\hline Smokers & 0.604 & .001 & 0.624 & 0.001 & 0.205 & .005 & 0.208 & .005 & 0.199 & .005 & 0.169 & .006 \\
\hline Sex & 0.000 & .054 & 0.000 & 0.040 & 0.162 & .006 & 0.923 & .000 & 0.954 & .000 & 0.415 & .002 \\
\hline
\end{tabular}


disease (CVD), insulin resistance (ID), and type 2 diabetes (T2D) have become even more critical with the increase in obesity (9). In some cases, an imbalance occurs between the ROS and/or free radicals and the antioxidant system. This condition called oxidative stress plays an important role in the etiology of obesity, T2D, and CVD. Many studies have reported that obesity increases oxidative stress, which is associated with the formation of fat tissue contributing to the development of obesity and metabolic syndrome (10)

The present study was novel in evaluating the relationship between dynamic thiol/disulfide homeostasis in the serum of patients and BMI using new, automated, calorimetric methods and values of native thiol, total thiol, disulfide, disulfide/native thiol, disulfide/total thiol, and native/total thiol. Some recent studies explored thiol/disulfide hemostasis. Üstüner et al. investigated native thiol, total thiol, and disulfide blood levels in 30 healthy controls and 30 patients with vitiligo. They found an increase in disulfide/native thiol and disulfide/total thiol values and blood disulfide levels, indicating thiol/disulfide imbalance in vitiligo as a result of oxidative stress and tissue inflammation. In addition, the levels of disulfide and total thiol strongly correlated with the severity of vitiligo (11).

Kundi et al. investigated the role of thiol/disulfide homeostasis in the presence of slow coronary flow. They found significant differences in thiol/disulfide homeostasis and slow coronary flow among the normal-flow groups. The results of the study showed that the high-sensitivity C-reactive protein and thiol/ disulfide ratio were independently associated with the slow coronary flow (12).

Ates et al. performed a study on 125 (54 male and 71 female) healthy volunteers and 125 (54 male and 71 female) prediabetic patients, who were aged more than 18 years and had not received any treatment earlier. They investigated the changes in the native thiol/disulfide ratio in both groups using the automatic measurement method developed by Erel and Neselioğlu. The native thiol $(P<0.001)$, total thiol $(P=0.008)$, and native thiol/total thiol $(P=0.022)$ values were lower, while the disulfide, disulfide/native thiol, and disulfide/total thiol values were higher, in prediabetic patients compared with controls. Further, a positive correlation was detected between disulfide and fasting blood glucose levels and glycated hemoglobin $(\mathrm{HbA} 1 \mathrm{c})$. This study showed that thiotoxidation increased in prediabetic patients, and a positive correlation was found between disulfide and blood glucose and $\mathrm{HbA} 1 \mathrm{c}$ levels (13).

In the present study, native thiol and total thio values were found to be higher in patients with low BMI. However, disulfide, disulfide/native thiol, disulfide/total thiol, and native/total thiol values were found to be significantly higher with the increase in $\mathrm{BMI}$, in parallel with the increase in oxidative stress. No significant difference was found in the thiol values of the smokers and nonsmokers.

\section{CONCLUSIONS}

It is predicted that thiol/disulfide homeostasis measurements can be effectively used, especially while choosing between medical and/or surgical treatment of obese patients because these measurements are cheap and easily applicable and accessible. Also, they can evaluate reduced oxidative stress in parallel with weight loss.

\section{REFERENCES}

1. Ates, N. Ozkayar, C. Topcuoglu, F. Dede. Relationship between oxidative stress parameters and asymptomatic organ damage in hypertensive patients without diabetes mellitus. Scand. Cardiovasc 2015;49:249-256.

2. Cremers C.M, Jakob U, Oxidant sensing by reversible disulfide bond formation. J. Biol. Chem 2013;288:26489-26496.

3. Schafer F.Q, Buettner G.R. Redox environment of the cell as viewed through the redox state of the glutathione disulfide/ glutathione couple. Free Radic. Biol. Med 2001;30:1191-1212.

4. Rodrigues S.D, Batista G.B, Ingberman M, Pecoits-Filho R Nakao L.S. Plasma cysteine/cystine reduction potentia correlates with plasma creatinine levels in chronic kidney disease. Blood Purif 2012;34:231-237.

5. Vaziri N.D. Causal link between oxidative stress, inflammation and hypertension. Iran. J. Kidney Dis 2008;2:1-10.

6. Turell L, Radi R, Alvarez B. The thiol pool in human plasma: the central contribution of albumin to redox processes. Free Radic Biol Med. 2013;65:244-253.

7. Jones DP, Liang Y. Measuring the poise of thiol/disulfide couples in vivo. Free Radic Biol Med 2009;47:1329-1338.

8. Biswas S, Chida AS, Rahman I. Redox modifications of proteinthiols: emerging roles in cell signaling. Biochem Pharmacol 2006;71:551-564.

9. Avignon A, Hokayem, Bisbal C, Lambert K. Dietary antioxidants: do they have a role to play in the on going fight against abnormal glucose metabolism? Nutrition. 2012;28:715-721.

10. Emekli N, Yiğitbaşı T. Klinik biyokimya. 1. Baskı, İstanbul: Akademi Ajans Matbaa; 2015

11. Üstüner P. The Role of Thiol/Disulfide Homeostasis in Vitiligo: A Novel Inflammatory Marker. IKSST Derg 2018;10:18-24

12. Kundi $\mathrm{H}$, Gok M, Cetin M et al. Association of thiol disulfide homeostasis with slow coronary flow. Scandinavian Cardiovascular Journal 2016;50:213-217

13. Ates I, Kaplan M, Inan B et al. How does thiol/disulfide homeostasis change in prediabetic patients?. Diabetes research and clinical practice 2015;110:166 - 171. 